Article

\title{
Synthesis and Biological Evaluation of Novel Benzothiazole-2-thiol Derivatives as Potential Anticancer Agents
}

\author{
Xuan-Hong Shi, Zhao Wang, Yong Xia, Ting-Hong Ye, Mei Deng, You-Zhi Xu, Yu-Quan Wei \\ and Luo-Ting $\mathrm{Yu}$ *
}

State Key Laboratory of Biotherapy, West China Hospital, West China Medical School, Sichuan University, Chengdu 610041, China

* Author to whom correspondence should be addressed; E-Mail: yuluot@scu.edu.cn; Tel: +86-28-8550-3817; Fax: +86-28-8516-4060.

Received: 22 February 2012; in revised form: 20 March 2012 / Accepted: 26 March 2012 /

Published: 30 March 2012

\begin{abstract}
A series of novel benzothiazole-2-thiol derivatives were synthesized and their structures determined by ${ }^{1} \mathrm{H}-\mathrm{NMR},{ }^{13} \mathrm{C}-\mathrm{NMR}$ and HRMS (ESI). The effects of all compounds on a panel of different types of human cancer cell lines were investigated. Among them, pyridinyl-2-amine linked benzothiazole-2-thiol compounds 7d, 7e, $7 \mathbf{f}$ and $7 \mathbf{i}$ exhibited potent and broad-spectrum inhibitory activities. Compound 7e displayed the most potent anticancer activity on SKRB-3 $\left(\mathrm{IC}_{50}=1.2 \mathrm{nM}\right)$, SW620 $\left(\mathrm{IC}_{50}=4.3 \mathrm{nM}\right)$, A549 $\left(\mathrm{IC}_{50}=44 \mathrm{nM}\right)$ and HepG2 $\left(\mathrm{IC}_{50}=48 \mathrm{nM}\right)$ and was found to induce apoptosis in HepG2 cancer cells.
\end{abstract}

Keywords: anticancer; synthesis; apoptosis; benzothiazole-2-thiol derivatives

\section{Introduction}

A number of benzothiazole derivatives have exhibited interesting biological activities [1-3] and attracted continuing interest for further molecular exploration as useful anticancer agents [4,5]. Our preceding studies had found that two benzothiazole-2-thiol compounds (compounds $\mathbf{1}$ and 2) displayed good anticancer activities and induced HepG2 cell apoptosis in vitro [6]. In order to develop more potent tumor growth inhibitors as novel anticancer agents, we designed and synthesized a series of novel benzothiazole-2-thiol derivatives through incorporation of heterocyclic rings (pyridine, pyrimidine and thiazole) to benzothiazole-2-thiol derivatives with the activity and safety advantages of the 
heterocyclic ring structures $[7,8]$. The effects of all the novel compounds on a panel of different types of human cancer cell lines were investigated by the MTT assay and compound 7e was selected to examine apoptosis on HepG2 cell cells by flow cytometry. As a result, the pyridinyl-2-amine linked benzothiazole-2-thiol compounds exhibited potent anticancer activities and compound 7e inhibited the proliferation of HepG2 cell via inducing apoptosis.

\section{Results and Discussion}

\subsection{Chemistry}

Twenty novel benzothiazole-2-thiol derivatives linked with heterocyclic rings were designed and synthesized by the route shown in Scheme 1.

Scheme 1. Synthetic route for $7 \mathbf{a}-\mathbf{t}$.

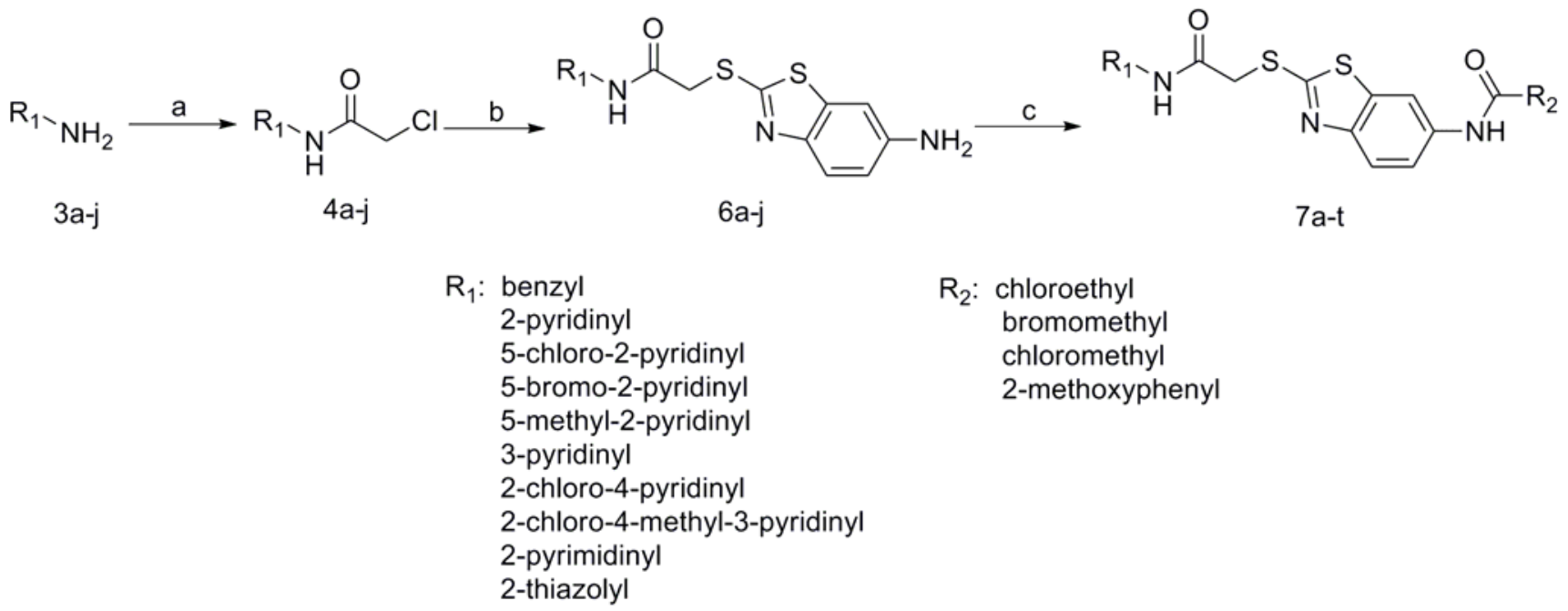

Reagents and conditions: (a) 2-chloroacetyl chloride, $\mathrm{CH}_{2} \mathrm{Cl}_{2}, \mathrm{~K}_{2} \mathrm{CO}_{3}, 4 \mathrm{~h}, \mathrm{rt}, 85 \%-93 \%$; (b) 6-aminobenzothiazole-2-thiol (compound 5), THF, $\mathrm{Et}_{3} \mathrm{~N}$, reflux, 4-6 h; (c) 2-methoxybenzoyl chloride/2-chloroacetyl chloride/3-chloropropanoyl chloride/2-bromoacetyl bromide, $\mathrm{Et}_{3} \mathrm{~N}, \mathrm{THF}, \mathrm{rt}$, $4-12$ h, 63\%-91\%.

Commercially available amines (compounds $\mathbf{3 a}-\mathbf{j}$ ) were first reacted with 2-chloroacetyl chloride in the presence of potassium carbonate as the base in dichloromethane to give crude compounds $\mathbf{4 a - j}$. The raw products $\mathbf{4 a - j}$ were purified by recrystallization from ethyl acetate/petroleum ether. The compounds $\mathbf{6 a}-\mathbf{j}$ were prepared by reacting $\mathbf{4 a - j}$ with 6-aminobenzothiazole-2-thiol (compound $\mathbf{5}$ ) and triethylamine (TEA) as the base in tetrahydrofuran (THF) under reflux. Compounds $\mathbf{6 a}-\mathbf{j}$ respectively were thus obtained and could be used directly for the next step without further purification. The reaction mixtures of compounds $\mathbf{6 a}-\mathbf{j}$ were further reacted with 3-chloropropyl chloride, 2-bromoacetyl bromide, 2-chloroacetyl chloride and 2-methoxybenzoyl chloride in the presence of triethylamine (TEA), respectively. The precipitates were collected by filtration and washed with water to yield the crude products (compounds $7 \mathbf{a}-\mathbf{t}$ ). Each compound was purified by column chromatography on silica gel using petroleum ether/ethyl acetate as eluent. The structures of all compounds were determined by ${ }^{1} \mathrm{H}-\mathrm{NMR},{ }^{13} \mathrm{C}-\mathrm{NMR}$ and HRMS (ESI). 


\subsection{Biological Activities}

The twenty novel synthesized benzothiazole-2-thiol derivatives were investigated for anticancer activity in vitro on cancer cell lines by the MTT assay with compounds $\mathbf{1}$ and $\mathbf{2}$ as positive controls. Compounds 1 and $\mathbf{2}$ showed good activity against human colon adenocarcinoma cell line (SW480), human cervical cancer cell line (HeLa) and human hepatocellular carcinoma cell line (HepG2) in our preceding studies. Herein, we selected firstly the three human cell lines to test these compounds and the results were presented in Table 1.

Table 1. The anti-proliferative activities for compounds $\mathbf{1 , 2}$ and $\mathbf{7 a - t .}$
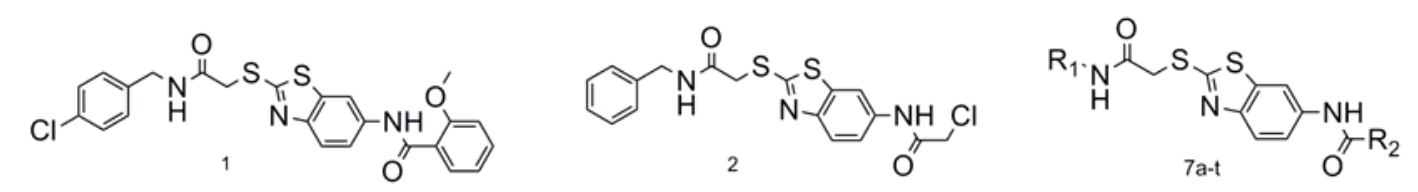

\begin{tabular}{llllll}
\hline \multirow{2}{*}{ Compd. } & \multirow{2}{*}{$\mathbf{R}_{\mathbf{1}}$} & \multirow{2}{*}{$\mathbf{R}_{\mathbf{2}}$} & \multicolumn{3}{c}{$\mathbf{I C}_{\mathbf{5 0}}(\boldsymbol{\mu M})^{\mathbf{a}}$} \\
\cline { 4 - 6 } & & 2-methoxyphenyl & 0.7 & 5.6 & 4.0 \\
\hline $\mathbf{1}$ & 4-chlorobenzyl & chloromethyl & 1.0 & 5.2 & 4.6 \\
$\mathbf{2}$ & benzyl & chloroethyl & 5.6 & 15.7 & 8.6 \\
$\mathbf{7 a}$ & benzyl & bromoethyl & 6.6 & 14.9 & 48.1 \\
$\mathbf{7 b}$ & benzyl & chloromethyl & 0.7 & 2.3 & 3.1 \\
$\mathbf{7 c}$ & 2-pyridinyl & chloromethyl & 0.26 & 0.46 & 0.035 \\
$\mathbf{7 d}$ & 5-chloro-2-pyridinyl & chloromethyl & 0.048 & 0.68 & 0.02 \\
$\mathbf{7 e}$ & 5-bromo-2-pyridinyl & chloromethyl & 0.091 & 1.0 & 0.03 \\
$\mathbf{7 f}$ & 5-methyl-2-pyridinyl & chloromethyl & 6.6 & 6.4 & 12.6 \\
$\mathbf{7 g}$ & 3-pyridinyl & chloromethyl & 5.0 & 2.9 & 1.9 \\
$\mathbf{7 h}$ & 2-chloro-4-pyridinyl & chloromethyl & 0.4 & 2.4 & 0.4 \\
$\mathbf{7 i}$ & 2-chloro-4-methyl-3-pyridinyl & chloromethyl & 24.3 & 54.8 & 24.3 \\
$\mathbf{7 j}$ & 2-pyrimidinyl & chloromethyl & 13.1 & 7.1 & 14.5 \\
$\mathbf{7 k}$ & 2-thiazolyl & 2-methoxyphenyl & $>100$ & $>100$ & $>100$ \\
$\mathbf{7 1}$ & 2-pyridinyl & 2-methoxyphenyl & $>100$ & $>100$ & 0.6 \\
$\mathbf{7 m}$ & 5-chloro-2-pyridinyl & 2-methoxyphenyl & 23.5 & 23.8 & 23.5 \\
$\mathbf{7 n}$ & 5-bromo-2-pyridinyl & 2-methoxyphenyl & 45.6 & 31.1 & 0.8 \\
$\mathbf{7 0}$ & 5-methyl-2-pyridinyl & 2-methoxyphenyl & $>100$ & $>100$ & $>100$ \\
$\mathbf{7 p}$ & 3-pyridinyl & 2-methoxyphenyl & $>100$ & $>100$ & $>100$ \\
$\mathbf{7 q}$ & 2-chloro-4-pyridinyl & 2-methoxyphenyl & 23.1 & $>100$ & 34.6 \\
$\mathbf{7 r}$ & 2-chloro-4-methyl-3-pyridinyl & 2-methoxyphenyl & 27.2 & $>100$ & $>100$ \\
$\mathbf{7 s}$ & 2-pyrimidyl & 2-methoxyphenyl & $>100$ & $>100$ & $>100$ \\
$\mathbf{7 t}$ & 2-thiazolyl & & & &
\end{tabular}

${ }^{\mathrm{a}}$ Values are means of three experiments.

These novel compounds showed great variation of $\mathrm{IC}_{50}$ values on the three cell lines and compounds $\mathbf{7 d}, \mathbf{7 e}, \mathbf{7 f}$ and $\mathbf{7 i}$ exhibited potent inhibitory activities. To further study the cytotoxic profile, the potent analogues $\mathbf{7 d}, \mathbf{7 e}, \mathbf{7} \mathbf{f}$ and $7 \mathbf{i}$ were selected for further evaluation of inhibitory activities against other eleven types of human cancer cell lines, including colon cancer cell lines HCT-116 and SW620, lung cancer cell line A549, prostate cancer cell line PC-3, pancreatic cancer cell 
line BxPC-3, breast cancer cell line BT474, epidermoid cancer cell line A431, ovarian cancer cell line SKOV-3, non-small cell lung cancer cell line H460, breast cancer cell line MDA-MB-468 and SKRB-3.

As shown in Table 2, compounds 7d, 7e, 7f and $7 \mathbf{i}$ exhibited potent and broad-spectrum anticancer activities which were much better than compounds $\mathbf{1}$ and $\mathbf{2}$. Among them, compound $\mathbf{7 d}$ showed the most potent antitumor activities against $\mathrm{A} 431\left(\mathrm{IC}_{50}=20 \mathrm{nM}\right)$ and compound 7e displayed the most potent anticancer activity on SKRB-3 $\left(\mathrm{IC}_{50}=1.2 \mathrm{nM}\right), \mathrm{SW} 620\left(\mathrm{IC}_{50}=4.3 \mathrm{nM}\right), \mathrm{A} 549\left(\mathrm{IC}_{50}=44 \mathrm{nM}\right)$ and HepG2 $\left(\mathrm{IC}_{50}=48 \mathrm{nM}\right)$. The antitumor activities of compounds $\mathbf{7 d}$ and $\mathbf{7 e}$ were about 10-1,000 times greater than compounds 1 and 2 (SW620, A549, SKRB-3 and HepG2). These results suggested that pyridin-2-amine linking benzothiazole-2-thiol derivatives have potent and broad-spectrum anti-cancer activities and be worth being further investigated as candidate of anticancer agent.

Table 2. The anti-proliferative activities for compounds $\mathbf{7 d}, \mathbf{7 e}, \mathbf{7 f}, \mathbf{7 i}, \mathbf{1}$ and $\mathbf{2}$ against various cancer cell lines.
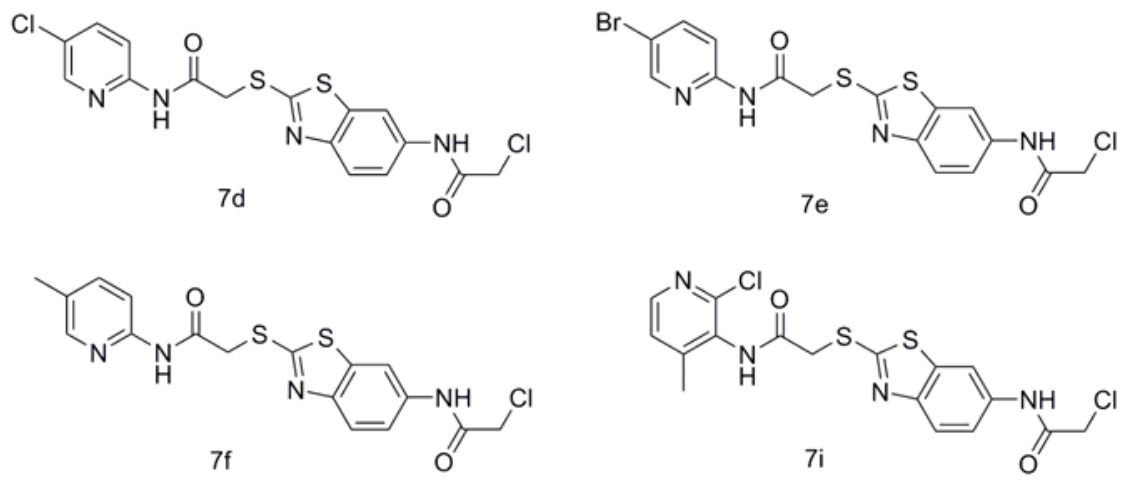

\begin{tabular}{|c|c|c|c|c|c|c|c|c|c|c|c|}
\hline \multirow[b]{2}{*}{ Compd. } & \multicolumn{11}{|c|}{$\mathrm{IC}_{50}(\mu \mathrm{M})^{\mathrm{a}}$} \\
\hline & HCT116 & BT474 & SW620 & H460 & PC-3 & BXPC-3 & A431 & A549 & SKOV-3 & $\begin{array}{l}\text { MDA- } \\
\text { MB- } \\
468 \\
\end{array}$ & SKRB-3 \\
\hline $7 d$ & 0.8 & 4.3 & 0.033 & 3.7 & 7.9 & 0.1 & 0.02 & 0.2 & 2.7 & 1.5 & 0.5 \\
\hline $7 e$ & 0.3 & 0.6 & 0.0043 & 0.3 & 6.0 & 0.3 & 0.2 & 0.044 & 0.4 & 0.9 & 0.0012 \\
\hline $7 f$ & 0.6 & 5.3 & 1.5 & 4.2 & 6.8 & 0.5 & 0.1 & 0.3 & 3.5 & 2.5 & 1.9 \\
\hline $7 \mathbf{i}$ & 2.2 & 5.2 & 6.5 & 14.6 & 8.6 & 9.3 & 4.8 & 8.3 & 5.8 & 6.8 & 11.3 \\
\hline 1 & 1.6 & $>100$ & 4.1 & 39.7 & 4.8 & 4.7 & 1.0 & 2.0 & 6.0 & 2.4 & 1.5 \\
\hline 2 & 1.2 & 7.8 & 1.3 & 10.0 & 6.0 & 6.6 & 4.5 & 4.4 & 4.6 & 4.0 & 8.0 \\
\hline
\end{tabular}

${ }^{\mathrm{a}}$ Values are means of three experiments.

In order to investigate the apoptosis effects of the benzothiazole-2-thiol derivatives, we selected compound 7e to examine apoptosis effects on HepG2 cells by flow cytometry. Flow cytometric analysis was performed to measure the apoptotic cells and the cell cycle after propidium iodide (PI) staining [9]. From Figure 1, the percentages of apoptotic cells were 34.2\%, 46.2\% and 53.3\%, respectively, with $0.625 \mu \mathrm{M}, 1.25 \mu \mathrm{M}$, and $2.5 \mu \mathrm{M}$ compound $7 \mathrm{e}$ treatment for $24 \mathrm{~h}$. The results indicated that compound 7e inhibited the proliferation of HepG2 cell via inducing apoptosis on a concentration-dependent manner. The exact and further biological mechanism of compound $7 \mathbf{e}$ is under investigation in our laboratory. 
Figure 1. Compound 7e concentration-dependently induced apoptosis in HepG2 cancer cells.
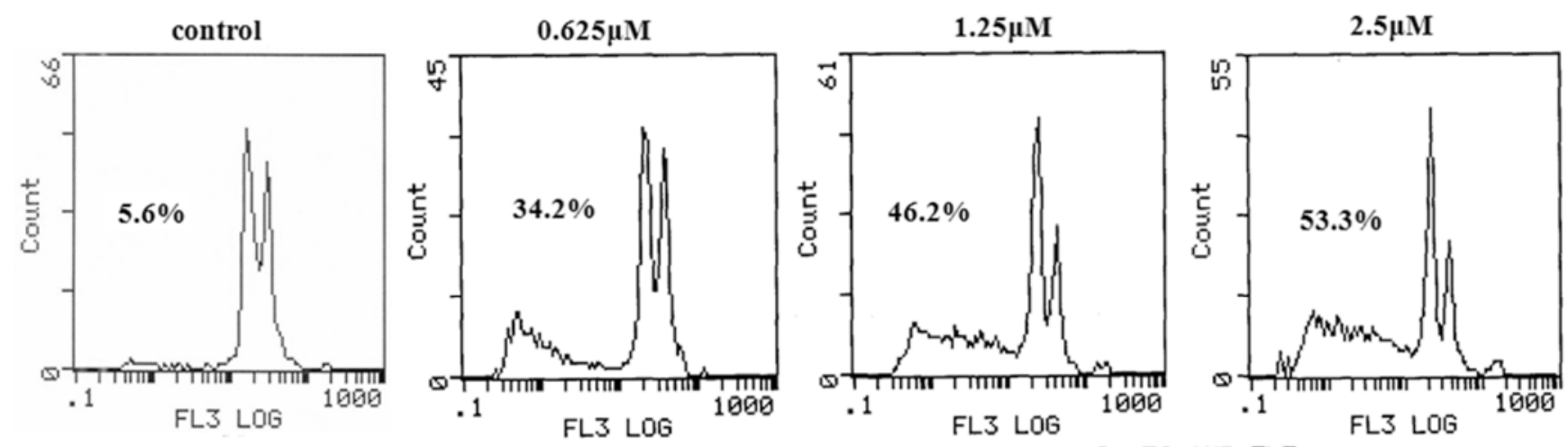

\section{Experimental}

\subsection{General}

The human cancer cell lines were purchased from the American Type Culture Collection (ATCC, Rockville, MD, USA). Dulbecco's modified Eagle medium (DMEM) and RPMI 1640 were purchased from Gibco (Grand Island, NY, USA). Fetal bovine serum (FBS) was purchased from Hyclone (Logan, UT, USA). Column chromatography was carried out on silica gel (200-300 mesh, Qingdao Marine Chemical Ltd., Qingdao, China). Thin layer chromatography (TLC) was performed on TLC silica gel 60 F254 plates. Melting points were measured using a on a Kofler hot stage apparatus and were uncorrected. ${ }^{1}$ H-NMR spectroscopy was performed using a Varian Unity Inova-400 spectrometer. The chemical shift values are reported in $\mathrm{d}$ units (ppm) relative to internal standard tetramethylsilane (TMS). ${ }^{13} \mathrm{C}-\mathrm{NMR}$ spectra were recorded on a Bruker AV II-600 MHz spectrometer. Mass spectrometry was carried out on a Waters Q-TOF Premier mass spectrometer. All solvents were dried and freshly distilled prior to use according to standard procedures. All the chemicals used were of analytical grade and commercially available. The purity of compound screened in biological assays was determined to be $\geq 90 \%$ by HPLC analysis with a photodiode array detector (Waters, Milford, MA, USA). An Atlantis $\mathrm{C}_{18}(150 \mathrm{~mm} \times 4.6 \mathrm{~mm}$, i.d. $5 \mu \mathrm{m})$ (Waters) was used with a gradient elution of methanol and HPLC-grade water as mobile phase at a flow rate of $1 \mathrm{~mL} / \mathrm{min}$.

\subsection{Preparation of Compounds $\mathbf{4 a - j}$}

Compounds $4 \mathbf{a}-\mathbf{j}$ were prepared following the literature procedure [10]. 2-Chloroacetyl chloride $(0.75 \mathrm{~mol})$ was added dropwise to a mixture of $\mathbf{3 a}-\mathbf{j}(0.5 \mathrm{~mol})$ and potassium carbonate $(1.0 \mathrm{~mol})$ in dichloromethane $(550 \mathrm{~mL})$ at $0{ }^{\circ} \mathrm{C}$ with stirring. After removal of the dichloromethane and vacuum filtration, the solid was washed with water and dried under vacuum for $12 \mathrm{~h}$ at $25-30{ }^{\circ} \mathrm{C}$. The title compounds $\mathbf{4 a}-\mathbf{j}$ were purified by recrystallization with petroleum ether/ethyl acetate.

\subsection{Preparation of Compounds $\mathbf{6 a}-\mathbf{j}$}

Compounds $\mathbf{6 a}-\mathbf{j}$ were synthesized according to a literature method [11] with some modifications. Briefly, 6-aminobenzothiazole-2-thiol $(35.0 \mathrm{mmol})$ was added to a mixture of $\mathbf{4 a}-\mathbf{j}(38.5 \mathrm{mmol})$ and 
triethylamine (TEA, $70.0 \mathrm{mmol}$ ) in tetrahydrofuran (THF, $480 \mathrm{~mL}$ ) at room temperature with stirring for $5 \mathrm{~h}$. The title compounds $\mathbf{6 a - j}$ were obtained, respectively, and could be used directly for the next step without further purification.

\subsection{General Procedure for Preparing Compounds 7a-t}

The reaction mixtures of compounds $\mathbf{6 a}-\mathbf{j}$ ( $3 \mathrm{mmol})$ were further reacted with acyl chloride $(3.6 \mathrm{mmol})$ in the presence of triethylamine (TEA, $4.5 \mathrm{mmol}$ ) using tetrahydrofuran (THF, $30 \mathrm{~mL}$ ) as solvent. The completion of the reaction was monitored by TLC and took 4-26 h. The precipitate was collected by filtration and washed with water to yield the crude product. Compounds $\mathbf{7} \mathbf{a}-\mathbf{t}$ were purified by column chromatography on silica gel using petroleum ether/ethyl acetate as eluent.

N-(2-(2-(Benzylamino)-2-oxoethylthio)benzo[d]thiazol-6-yl)-3-chloropropanamide (7a). Yellow powder, yield $72 \%$, purity $99.8 \%$, mp $177.2-178.1{ }^{\circ} \mathrm{C} ;{ }^{1} \mathrm{H}-\mathrm{NMR}$ (DMSO- $\left.d_{6}\right) \delta: 2.87(\mathrm{t}, J=6.2 \mathrm{~Hz}, 2 \mathrm{H}$ ), 3.91 (t, $J=6.2 \mathrm{~Hz}, 2 \mathrm{H}), 4.19(\mathrm{~s}, 2 \mathrm{H}), 4.33(\mathrm{~d}, J=6.0 \mathrm{~Hz}, 2 \mathrm{H}), 7.22-7.28(\mathrm{~m}, 5 \mathrm{H}), 7.55(\mathrm{dd}, J=2.0,4.4 \mathrm{~Hz}, 1 \mathrm{H})$, $7.77(\mathrm{~d}, J=8.8 \mathrm{~Hz}, 1 \mathrm{H}), 8.40(\mathrm{~d}, J=2.0 \mathrm{~Hz}, 1 \mathrm{H}), 8.82(\mathrm{t}, J=5.8 \mathrm{~Hz}, 1 \mathrm{H}), 10.33(\mathrm{~s}, 1 \mathrm{H})$; ${ }^{13} \mathrm{C}-\mathrm{NMR}\left(\mathrm{DMSO}-d_{6}\right) \delta: 36.53,39.49,40.76,42.54,111.35,118.49,121.04,126.76,127.10,128.19$, 131.70, 135.45, 135.81, 138.80, 148.68, 148.80, 164.35, 166.43, 168.09; HRMS (ESI) $\mathrm{m} / z$ : Calcd. for $\mathrm{C}_{19} \mathrm{H}_{18} \mathrm{ClN}_{3} \mathrm{O}_{2} \mathrm{~S}_{2}$ 420.0602; Found: $420.0592\left(\mathrm{M}-\mathrm{H}^{+}\right)$.

N-Benzyl-2-(6-(2-bromoacetamido)benzo[d]thiazol-2-ylthio)acetamide (7b). Yellow powder, yield 75\%, purity 98.0\%, mp 155.6-159.7 ${ }^{\circ} \mathrm{C}$; ${ }^{1} \mathrm{H}-\mathrm{NMR}$ (DMSO-d $) \delta$ : 4.08 (s, 2H), $4.20(\mathrm{~s}, 2 \mathrm{H}), 4.33$ (d, $J=6.0 \mathrm{~Hz}, 2 \mathrm{H}), 7.22-7.28(\mathrm{~m}, 5 \mathrm{H}), 7.55(\mathrm{~d}, J=8.4 \mathrm{~Hz}, 1 \mathrm{H}), 7.79(\mathrm{~d}, J=8.8 \mathrm{~Hz}, 1 \mathrm{H}), 8.38(\mathrm{~s}, 1 \mathrm{H})$, $8.83(\mathrm{~s}, 1 \mathrm{H}), 10.64(\mathrm{~s}, 1 \mathrm{H}) ;{ }^{13} \mathrm{C}-\mathrm{NMR}\left(\mathrm{DMSO}-d_{6}\right) \delta: 28.72,39.48,42.54,111.56,118.65,121.10$, 126.86, 127.32, 128.29, 131.86, 135.48, 135.59, 138.94, 148.74, 148.81, 165.23, 167.36, 169.70; HRMS (ESI) $m / z$ : Calcd. for $\mathrm{C}_{18} \mathrm{H}_{16} \mathrm{BrN}_{3} \mathrm{O}_{2} \mathrm{~S}_{2} 449.9940$; Found: $449.9929\left(\mathrm{M}-\mathrm{H}^{+}\right)$.

2-Chloro-N-(2-(2-oxo-2-(pyridin-2-ylamino)ethylthio)benzo[d]thiazol-6-yl)acetamide (7c). Yellow powder, yield $70 \%$, purity $92.0 \%$, mp $168.4-172.9{ }^{\circ} \mathrm{C} ;{ }^{1} \mathrm{H}-\mathrm{NMR}$ (DMSO- $\left.d_{6}\right) \delta$ : $4.29(\mathrm{~s}, 2 \mathrm{H}), 4.45(\mathrm{~s}, 2 \mathrm{H}$ ), $7.54-7.58(\mathrm{~m}, 2 \mathrm{H}), 7.78$ (d, $J=8.8 \mathrm{~Hz}, 1 \mathrm{H}), 8.18-8.19(\mathrm{~m}, 2 \mathrm{H}), 8.37$ (d, $J=16.0 \mathrm{~Hz}, 1 \mathrm{H}), 8.89$ $(\mathrm{s}, 1 \mathrm{H}), 10.35(\mathrm{~s}, 1 \mathrm{H}), 10.54(\mathrm{~s}, 1 \mathrm{H}) ;{ }^{13} \mathrm{C}-\mathrm{NMR}$ (DMSO-d $) \delta: 39.46,43.49,111.69,113.46,118.75$, 119.69, 121.12, 135.42, 135.57, 138.32, 148.02, 148.91, 151.59, 164.63, 164.79, 166.20; HRMS (ESI) $m / z$ : Calcd. for $\mathrm{C}_{16} \mathrm{H}_{13} \mathrm{ClN}_{4} \mathrm{O}_{2} \mathrm{~S}_{2}$ 393.0241; Found: $393.0241\left(\mathrm{M}-\mathrm{H}^{+}\right)$.

2-Chloro-N-(2-(2-(5-chloropyridin-2-ylamino)-2-oxoethylthio)benzo[d]thiazol-6-yl)acetamide (7d). Yellow powder, yield $70 \%$, purity $96.8 \%$, mp $217.2-218.8{ }^{\circ} \mathrm{C} ;{ }^{1} \mathrm{H}-\mathrm{NMR}$ (DMSO- $\left.d_{6}\right) \delta: 4.29$ (s, 2H), $4.44(\mathrm{~s}, 2 \mathrm{H}), 7.50-7.54(\mathrm{~m}, 1 \mathrm{H}), 7.77(\mathrm{~d}, J=8.8 \mathrm{~Hz}, 1 \mathrm{H}), 8.41(\mathrm{~s}, 1 \mathrm{H}), 7.50-8.41(\mathrm{~m}, 3 \mathrm{H}), 10.54(\mathrm{~s}, 1 \mathrm{H})$, $11.10(\mathrm{~s}, 1 \mathrm{H}) ;{ }^{13} \mathrm{C}-\mathrm{NMR}$ (DMSO- $\left.d_{6}\right) \delta: 39.48,43.54,111.80,114.52,118.77,121.16,125.38,135.49$, 135.61, 138.01, 148.79, 148.94, 150.27, 164.62, 165.37, 166.35; HRMS (ESI) $m / z$ : Calcd. for $\mathrm{C}_{16} \mathrm{H}_{12} \mathrm{Cl}_{2} \mathrm{~N}_{4} \mathrm{O}_{2} \mathrm{~S}_{2}$ 426.9852; Found: $426.9822\left(\mathrm{M}-\mathrm{H}^{+}\right)$.

N-(5-Bromopyridin-2-yl)-2-(6-(2-chloroacetamido)benzo[d]thiazol-2-ylthio)acetamide (7e). Yellow powder, yield 71\%, purity 96.0\%, mp 221.3-222.5 ${ }^{\circ} \mathrm{C}$; ${ }^{1} \mathrm{H}-\mathrm{NMR}$ (DMSO- $d_{6}$ ) $\delta$ : $4.29(\mathrm{~s}, 2 \mathrm{H}), 4.43(\mathrm{~s}, 2 \mathrm{H})$, $7.53(\mathrm{~d}, J=8.8 \mathrm{~Hz}, 1 \mathrm{H}), 7.76(\mathrm{~d}, J=8.8 \mathrm{~Hz}, 1 \mathrm{H}), 8.47(\mathrm{~s}, 1 \mathrm{H}), 8.38(\mathrm{~s}, 1 \mathrm{H}), 8.02(\mathrm{~m}, 2 \mathrm{H}), 10.54(\mathrm{~s}, 1 \mathrm{H})$, 
$11.09(\mathrm{~s}, 1 \mathrm{H}) ;{ }^{13} \mathrm{C}-\mathrm{NMR}$ (DMSO- $\left.d_{6}\right) \delta: 39.98,44.05,112.29,114.26,115.56,119.26,121.66,135.79$, 135.99, 141.22, 149.12, 149.44, 151.07, 165.11, 165.23, 166.88; HRMS (ESI) $m / z$ : Calcd. for $\mathrm{C}_{16} \mathrm{H}_{12} \mathrm{BrClN}_{4} \mathrm{O}_{2} \mathrm{~S}_{2}$ 472.7864; Found: $472.7853\left(\mathrm{M}-\mathrm{H}^{+}\right)$.

2-Chloro-N-(2-(2-(5-methylpyridin-2-ylamino)-2-oxoethylthio)benzo[d]thiazol-6-yl)acetamide (7f). Yellow powder, yield 90\%, purity 97.1\%, mp 214.0-214.9 ${ }^{\circ} \mathrm{C} ;{ }^{1} \mathrm{H}-\mathrm{NMR}$ (DMSO- $d_{6}$ ) $\delta: 2.25$ (s, 3H), $4.29(\mathrm{~s}, 2 \mathrm{H}), 4.41(\mathrm{~s}, 2 \mathrm{H}), 7.53(\mathrm{dd}, J=2.0,4.4 \mathrm{~Hz}, 1 \mathrm{H}), 7.61(\mathrm{dd}, J=2.0,4.2 \mathrm{~Hz}, 1 \mathrm{H}), 7.77(\mathrm{~d}$, $J=9.2 \mathrm{~Hz}, 1 \mathrm{H}), 7.94(\mathrm{~d}, J=8.4 \mathrm{~Hz}, 1 \mathrm{H}), 8.18(\mathrm{~s}, 1 \mathrm{H}), 8.37$ (d, $J=1.0 \mathrm{~Hz}, 1 \mathrm{H}), 10.54(\mathrm{~s}, 1 \mathrm{H}), 10.82$ $(\mathrm{s}, 1 \mathrm{H}) ;{ }^{13} \mathrm{C}-\mathrm{NMR}\left(\mathrm{DMSO}-d_{6}\right) \delta: 17.22,39.46,43.53,111.75,113.13,118.74,121.14,128.73,135.30$, 135.47, 139.06,147.22, 148.95, 149.20, 164.70, 164.74, 166.02; HRMS (ESI) $\mathrm{m} / z$ : Calcd. for $\mathrm{C}_{17} \mathrm{H}_{15} \mathrm{ClN}_{4} \mathrm{O}_{2} \mathrm{~S}_{2}$ 407.0398; Found: $407.0397\left(\mathrm{M}-\mathrm{H}^{+}\right)$.

2-Chloro-N-(2-(2-oxo-2-(pyridin-3-ylamino)ethylthio)benzo[d]thiazol-6-yl)acetamide (7g). Yellow powder, yield 68\%, purity $92.0 \%$, mp $160.6-165.4{ }^{\circ} \mathrm{C} ;{ }^{1} \mathrm{H}-\mathrm{NMR}$ (DMSO- $\left.d_{6}\right) \delta$ : $4.29(\mathrm{~s}, 2 \mathrm{H}), 4.45(\mathrm{~s}, 2 \mathrm{H}$ ), $7.55(\mathrm{~d}, J=8.0 \mathrm{~Hz}, 2 \mathrm{H}), 7.78$ (d, $J=8.8 \mathrm{~Hz}, 1 \mathrm{H}), 8.19$ (d, $J=8.4 \mathrm{~Hz}, 1 \mathrm{H}), 8.37$ (s, 2H), 8.89 (s, 1H), $10.66(\mathrm{~s}, 1 \mathrm{H}), 11.20(\mathrm{~s}, 1 \mathrm{H}) ;{ }^{13} \mathrm{C}-\mathrm{NMR}\left(\mathrm{DMSO}-d_{6}\right) \delta: 39.43,43.50,111.67,118.52,118.72,121.18$, 130.46, 135.43, 135.51, 140.30, 148.83, 148.89, 164.36, 164.80, 166.47; HRMS (ESI) $\mathrm{m} / z$ : Calcd. for $\mathrm{C}_{16} \mathrm{H}_{13} \mathrm{ClN}_{4} \mathrm{O}_{2} \mathrm{~S}_{2}$ 393.0241; Found: $393.0247\left(\mathrm{M}-\mathrm{H}^{+}\right)$.

2-Chloro-N-(2-(2-(2-chloropyridin-4-ylamino)-2-oxoethylthio)benzo[d]thiazol-6-yl)acetamide (7h). Yellow powder, yield 91\%, purity 95.7\%, mp 192.5-197.1 ${ }^{\circ} \mathrm{C} ;{ }^{1} \mathrm{H}-\mathrm{NMR}$ (DMSO-d 6 ) $\delta: 4.29$ (s, 2H), $4.43(\mathrm{~s}, 2 \mathrm{H}), 7.47-7.54(\mathrm{~m}, 2 \mathrm{H}), 7.75-7.78(\mathrm{~m}, 2 \mathrm{H}), 8.29(\mathrm{~d}, J=5.6 \mathrm{~Hz}, 1 \mathrm{H}), 8.39(\mathrm{~s}, 1 \mathrm{H}), 10.55(\mathrm{~s}, 1 \mathrm{H})$, $11.08(\mathrm{~s}, 1 \mathrm{H}) ;{ }^{13} \mathrm{C}-\mathrm{NMR}$ (DMSO- $\left.d_{6}\right) \delta: 39.48,43.53,111.81,112.46,112.62,118.76,121.15,135.34$, 135.49, 147.83, 148.85, 150.47, 151.02, 164.34, 164.75, 166.93; HRMS (ESI) $\mathrm{m} / z$ : Calcd. for $\mathrm{C}_{16} \mathrm{H}_{12} \mathrm{Cl}_{2} \mathrm{~N}_{4} \mathrm{O}_{2} \mathrm{~S}_{2}$ 426.9852; Found: $426.9883\left(\mathrm{M}-\mathrm{H}^{+}\right)$.

2-Chloro-N-(2-(2-(2-chloro-4-methylpyridin-3-ylamino)-2-oxoethylthio)benzo[d]thiazol-6-yl)acetamide (7i). Yellow powder, yield $80 \%$, purity $98.0 \%$, mp 186.4-188.3 ${ }^{\circ} \mathrm{C} ;{ }^{1} \mathrm{H}-\mathrm{NMR}$ (DMSO- $d_{6}$ ) $\delta: 2.22(\mathrm{~s}, 3 \mathrm{H}$ ), 4.29 $(\mathrm{s}, 2 \mathrm{H}), 4.41(\mathrm{~s}, 2 \mathrm{H}), 7.34(\mathrm{~d}, J=4.8 \mathrm{~Hz}, 1 \mathrm{H}), 7.67(\mathrm{t}, J=8.8 \mathrm{~Hz}, 2 \mathrm{H}), 8.19(\mathrm{~d}, J=4.8 \mathrm{~Hz}, 1 \mathrm{H}), 8.55$ $(\mathrm{s}, 1 \mathrm{H}), 10.24(\mathrm{~s}, 1 \mathrm{H}), 10.37(\mathrm{~s}, 1 \mathrm{H}) ;{ }^{13} \mathrm{C}-\mathrm{NMR}\left(\mathrm{DMSO}-d_{6}\right) \delta: 17.81,39.54,43.53,111.87,118.83$, 121.08, 125.10, 130.49, 135.30, 135.56, 147.02, 148.67, 148.77, 148.99, 164.43, 164.76, 165.62; HRMS (ESI) $m / z$ : Calcd. for $\mathrm{C}_{17} \mathrm{H}_{14} \mathrm{Cl}_{2} \mathrm{~N}_{4} \mathrm{O}_{2} \mathrm{~S}_{2} 441.0008$; Found: $440.9992\left(\mathrm{M}-\mathrm{H}^{+}\right.$).

2-Chloro-N-(2-(2-oxo-2-(pyrimidin-2-ylamino)ethylthio)benzo[d]thiazol-6-yl)acetamide (7j). Brownish red powder, yield 63\%, purity $90.0 \%$, mp 172.6-174.9 ${ }^{\circ} \mathrm{C} ;{ }^{1} \mathrm{H}-\mathrm{NMR}$ (DMSO- $\left.d_{6}\right) \delta: 4.29(\mathrm{~s}, 2 \mathrm{H}), 4.59$ (s, 2H), 7.22 (t, $J=4.8 \mathrm{~Hz}, 1 \mathrm{H}), 7.54(\mathrm{~d}, J=4.4 \mathrm{~Hz}, 1 \mathrm{H}), 7.78(\mathrm{~d}, J=8.8 \mathrm{~Hz}, 1 \mathrm{H}), 8.38(\mathrm{~s}, 1 \mathrm{H}), 8.69$ $(\mathrm{d}, J=2.4 \mathrm{~Hz}, 2 \mathrm{H}), 10.58(\mathrm{~s}, 1 \mathrm{H}), 11.04(\mathrm{~s}, 1 \mathrm{H}) ;{ }^{13} \mathrm{C}-\mathrm{NMR}$ (DMSO-d $\left.)_{6}\right) \delta: 39.46,43.52,109.98$, 117.43, 121.60, 136.22, 147.29, 157.33, 157.66, 158.40, 166.22; HRMS (ESI) $m / z$ : Calcd. for $\mathrm{C}_{15} \mathrm{H}_{12} \mathrm{ClN}_{5} \mathrm{O}_{2} \mathrm{~S}_{2}$ 394.0194; Found: $394.0109\left({\left.\mathrm{M}-\mathrm{H}^{+}\right) .}^{+}\right.$

2-Chloro-N-(2-(2-oxo-2-(thiazol-2-ylamino)ethylthio)benzo[d]thiazol-6-yl)acetamide (7k). Yellow powder, yield 78\%, purity 95.0\%, mp 218.3-224.7 ${ }^{\circ} \mathrm{C}$; ${ }^{1} \mathrm{H}-\mathrm{NMR}$ (DMSO-d ${ }_{6}$ ) $\delta: 4.29$ (s, 2H), 4.46 (s, 2H), $7.25(\mathrm{~d}, J=3.6 \mathrm{~Hz}, 1 \mathrm{H}), 7.50-7.55(\mathrm{~m}, 2 \mathrm{H}), 7.77(\mathrm{~d}, J=8.8 \mathrm{~Hz}, 1 \mathrm{H}), 8.38(\mathrm{~s}, 1 \mathrm{H}), 10.55(\mathrm{~s}, 1 \mathrm{H})$, 
$12.53(\mathrm{~s}, 1 \mathrm{H}) ;{ }^{13} \mathrm{C}-\mathrm{NMR}$ (DMSO- $\left.d_{6}\right) \delta: 39.46,43.54,111.81,113.79,118.77,121.18,135.33,135.52$, 137.71, 148.87, 157.75, 164.32, 164.74, 165.64; HRMS (ESI) $m / z$ : Calcd. for $\mathrm{C}_{14} \mathrm{H}_{11} \mathrm{ClN}_{4} \mathrm{O}_{2} \mathrm{~S}_{3}$ 398.9806; Found: $398.9816\left(\mathrm{M}-\mathrm{H}^{+}\right)$.

2-Methoxy-N-(2-(2-oxo-2-(pyridin-2-ylamino)ethylthio)benzo[d]thiazol-6-yl)benzamide (71). Yellow powder, yield $70 \%$, purity $92.0 \%$, mp $154.2-158.9{ }^{\circ} \mathrm{C} ;{ }^{1} \mathrm{H}-\mathrm{NMR}$ (DMSO- $\left.d_{6}\right) \delta$ : $3.90(\mathrm{~s}, 3 \mathrm{H}), 4.45$ (s, 2H), $7.08(\mathrm{t}, J=7.4 \mathrm{~Hz}, 1 \mathrm{H}), 7.14(\mathrm{t}, J=6.0 \mathrm{~Hz}, 1 \mathrm{H}), 7.19(\mathrm{~d}, J=8.4 \mathrm{~Hz}, 1 \mathrm{H}), 7.49-7.54(\mathrm{~m}, 1 \mathrm{H})$, $7.63-7.69(\mathrm{~m}, 2 \mathrm{H}), 7.80(\mathrm{dd}, J=7.0,6.4 \mathrm{~Hz}, 2 \mathrm{H}), 8.35(\mathrm{~d}, J=3.6 \mathrm{~Hz}, 1 \mathrm{H}), 8.04(\mathrm{~d}, J=8.4 \mathrm{~Hz}, 1 \mathrm{H})$, $8.54(\mathrm{~s}, 1 \mathrm{H}), 10.35(\mathrm{~s}, 1 \mathrm{H}), 10.91(\mathrm{~s}, 1 \mathrm{H}) ;{ }^{13} \mathrm{C}-\mathrm{NMR}\left(\mathrm{DMSO}-d_{6}\right) \delta: 39.46,111.69,118.75,121.12$, 135.41, 135.96, 148.91, 164.19, 164.57, 166.22; HRMS (ESI) $m / z$ : Calcd. for $\mathrm{C}_{22} \mathrm{H}_{18} \mathrm{~N}_{4} \mathrm{O}_{3} \mathrm{~S}_{2} 451.0893$; Found: $451.0887\left(\mathrm{M}-\mathrm{H}^{+}\right)$.

N-(2-(2-(5-Chloropyridin-2-ylamino)-2-oxoethylthio)benzo[d] thiazol-6-yl)-2-methoxybenzamide (7m). Yellow powder, yield 70\%, purity 97.0\%, mp 148.5-154.1 ${ }^{\circ} \mathrm{C} ;{ }^{1} \mathrm{H}-\mathrm{NMR}$ (DMSO- $d_{6}$ ) $\delta: 3.90$ (s, 3H), 4.45 (s, 2H), 7.19 (d, $J=8.4 \mathrm{~Hz}, 1 \mathrm{H}), 7.2(\mathrm{t}, J=7.4 \mathrm{~Hz}, 2 \mathrm{H}), 7.63-7.75(\mathrm{~m}, 2 \mathrm{H}), 7.77(\mathrm{~d}, J=8.8 \mathrm{~Hz}, 1 \mathrm{H})$, $7.92(\mathrm{~d}, J=4.4 \mathrm{~Hz}, 1 \mathrm{H}), 8.08(\mathrm{~d}, J=8.4 \mathrm{~Hz}, 1 \mathrm{H}), 8.41(\mathrm{~d}, J=2.4 \mathrm{~Hz}, 1 \mathrm{H}), 8.54(\mathrm{~s}, 1 \mathrm{H}), 10.35(\mathrm{~s}, 1 \mathrm{H})$, $11.11(\mathrm{~s}, 1 \mathrm{H}) ;{ }^{13} \mathrm{C}-\mathrm{NMR}$ (DMSO- $\left.d_{6}\right) \delta: 39.46,55.84,111.83,111.93,114.52,119.17,120.46,120.99$, $124.68,125.38,129.66,132,09,135.41,135.95,137.99,146.43,148.73,150.28,156.46,164,17$,

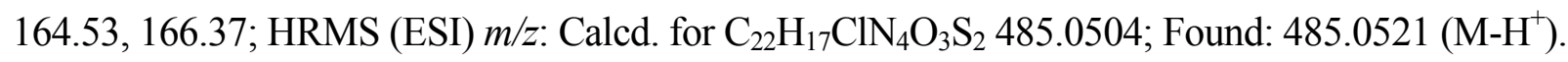

$N$-(2-(2-(5-Bromopyridin-2-ylamino)-2-oxoethylthio)benzo[d] thiazol-6-yl)-2-methoxybenzamide (7n). Yellow powder, yield $75 \%$, purity $99.0 \%$, mp 178.4-183.5 ${ }^{\circ} \mathrm{C} ;{ }^{1} \mathrm{H}-\mathrm{NMR}$ (DMSO- $d_{6}$ ) $\delta: 3.90$ (s, 3H), 4.45 (s, 2H), 7.07 (t, $J=7.4 \mathrm{~Hz}, 1 \mathrm{H}), 7.19(\mathrm{~d}, J=8.4 \mathrm{~Hz}, 1 \mathrm{H}), 7.51$ (t, $J=7.8 \mathrm{~Hz}, 1 \mathrm{H}), 7.66$ (d, $J=10.8 \mathrm{~Hz}, 2 \mathrm{H}), 7.77(\mathrm{~d}, J=8.8 \mathrm{~Hz}, 1 \mathrm{H}), 7.95-8.03(\mathrm{~m}, 2 \mathrm{H}), 8.48(\mathrm{~s}, 1 \mathrm{H}), 8.54(\mathrm{~s}, 1 \mathrm{H}), 10.35(\mathrm{~s}, 1 \mathrm{H})$, $11.10(\mathrm{~s}, 1 \mathrm{H}) ;{ }^{13} \mathrm{C}-\mathrm{NMR}$ (DMSO- $\left.d_{6}\right) \delta: 39.49,55.87,111.86,111.95,113.78,115.08,119.19,120.48$, $121.01,124.69$, 129.68, 132.11, 135.41, 135.96, 148.63, 148.74, 150.59, 156.48, 164.17, 164.54,

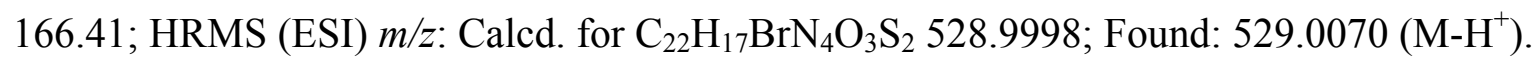

2-Methoxy-N-(2-(2-(5-methylpyridin-2-ylamino)-2-oxoethylthio)benzo[d]thiazol-6-yl)benzamide (7o). Yellow powder, yield $76 \%$, purity $92.0 \%$, mp 182.7-188.5 ${ }^{\circ} \mathrm{C} ;{ }^{1} \mathrm{H}-\mathrm{NMR}$ (DMSO- $d_{6}$ ) $\delta: 2.28$ (s, 3H), $3.90(\mathrm{~s}, 3 \mathrm{H}), 4.45(\mathrm{~s}, 2 \mathrm{H}), 7.08(\mathrm{t}, J=7.6 \mathrm{~Hz}, 1 \mathrm{H}), 7.19(\mathrm{~d}, J=8.4 \mathrm{~Hz}, 1 \mathrm{H}), 7.50-7.62(\mathrm{~m}, 2 \mathrm{H}), 7.68(\mathrm{t}$, $J=10.2 \mathrm{~Hz}, 2 \mathrm{H}), 7.78(\mathrm{~d}, J=8.8 \mathrm{~Hz}, 1 \mathrm{H}), 7.95(\mathrm{~s}, 1 \mathrm{H}), 8.18(\mathrm{~s}, 1 \mathrm{H}), 8.52(\mathrm{~d}, J=2.0 \mathrm{~Hz}, 1 \mathrm{H}), 10.31$ $(\mathrm{s}, 1 \mathrm{H}), 10.76(\mathrm{~s}, 1 \mathrm{H}) ;{ }^{13} \mathrm{C}-\mathrm{NMR}$ (DMSO- $\left.d_{6}\right) \delta: 17.24,39.49,55.86,111.84,111.96,112.99,119.18$, $120.47,121.00,124.71,128.61,129.67,132.10,135.41,135.95,138.57,147.80,148.79,149.49$, 156.48, 164.33, 164.55, 165.90; HRMS (ESI) $m / z$ : Calcd. for $\mathrm{C}_{23} \mathrm{H}_{20} \mathrm{~N}_{4} \mathrm{O}_{3} \mathrm{~S}_{2}$ 465.1050; Found: $465.1071\left(\mathrm{M}-\mathrm{H}^{+}\right)$.

2-Methoxy-N-(2-(2-oxo-2-(pyridin-3-ylamino)ethylthio)benzo[d]thiazol-6-yl)benzamide (7p). Yellow powder, yield $75 \%$, purity $95.0 \%$, mp $154.8-159.5{ }^{\circ} \mathrm{C}$; ${ }^{1} \mathrm{H}-\mathrm{NMR}$ (DMSO- $\left.d_{6}\right) \delta: 3.90(\mathrm{~s}, 3 \mathrm{H}), 4.45(\mathrm{~s}, 2 \mathrm{H})$, $7.08(\mathrm{t}, J=7.6 \mathrm{~Hz}, 1 \mathrm{H}), 7.19(\mathrm{~d}, J=8.4 \mathrm{~Hz}, 1 \mathrm{H}), 7.50-7.62(\mathrm{~m}, 2 \mathrm{H}), 7.55(\mathrm{~d}, J=8.0 \mathrm{~Hz}, 1 \mathrm{H}), 7.70(\mathrm{t}$, $J=6.6 \mathrm{~Hz}, 1 \mathrm{H}), 7.78(\mathrm{~d}, J=8.8 \mathrm{~Hz}, 1 \mathrm{H}), 8.29(\mathrm{~d}, J=8.4 \mathrm{~Hz}, 1 \mathrm{H}), 8.46(\mathrm{~d}, J=4.4 \mathrm{~Hz}, 1 \mathrm{H}), 8.39(\mathrm{~s}, 1 \mathrm{H})$, $8.95(\mathrm{~s}, 1 \mathrm{H}), 10.36(\mathrm{~s}, 1 \mathrm{H}), 11.12(\mathrm{~s}, 1 \mathrm{H}) ;{ }^{13} \mathrm{C}-\mathrm{NMR}\left(\mathrm{DMSO}-d_{6}\right) \delta: 39.44,55.62,111.90,112.33$, $119.21,119.95,120.99,121.24,124.69,126.23,129.67,132.97,135.41,135.96,140.75,144.51$, 
148.75, 156.47, 158.03, 164.19, 164.57, 167.32; HRMS (ESI) m/z: Calcd. for $\mathrm{C}_{22} \mathrm{H}_{18} \mathrm{~N}_{4} \mathrm{O}_{3} \mathrm{~S}_{2} 451.0893$; Found: $451.0833\left(\mathrm{M}-\mathrm{H}^{+}\right)$.

$N$-(2-(2-(2-Chloropyridin-4-ylamino)-2-oxoethylthio)benzo[d] thiazol-6-yl)-2-methoxybenzamide (7q). Yellow powder, yield 70\%, purity 90.0\%, mp 177.9-180.8 ${ }^{\circ} \mathrm{C} ;{ }^{1} \mathrm{H}-\mathrm{NMR}$ (DMSO- $d_{6}$ ) $\delta: 3.90$ (s, 3H), $4.44(\mathrm{~s}, 2 \mathrm{H}), 7.07$ (t, $J=7.4 \mathrm{~Hz}, 1 \mathrm{H}), 7.19(\mathrm{~d}, J=8.0 \mathrm{~Hz}, 1 \mathrm{H}), 7.47-7.53(\mathrm{~m}, 2 \mathrm{H}), 7.53-7.62(\mathrm{~m}, 2 \mathrm{H})$, $7.76(\mathrm{t}, J=5.8 \mathrm{~Hz}, 1 \mathrm{H}), 7.78(\mathrm{~s}, 1 \mathrm{H}), 8.29(\mathrm{~d}, J=5.6 \mathrm{~Hz}, 1 \mathrm{H}), 8.55(\mathrm{~s}, 1 \mathrm{H}), 10.35(\mathrm{~s}, 1 \mathrm{H}), 11.06(\mathrm{~s}, 1 \mathrm{H})$;

${ }^{13} \mathrm{C}-\mathrm{NMR}\left(\mathrm{DMSO}-d_{6}\right) \delta$ : 39.44, 55.84, 111.90, 111.93, 112.47, 112.62, 119.20, 120.46, 120.99, 124.67, 129.65, 132.11, 135.41, 135.97, 148.65, 150.47, 151.03, 156.45, 163.91, 164.57, 166.95; HRMS (ESI) $m / z$ : Calcd. for $\mathrm{C}_{22} \mathrm{H}_{17} \mathrm{ClN}_{4} \mathrm{O}_{3} \mathrm{~S}_{2}$ 485.0504; Found: $485.0491\left(\mathrm{M}-\mathrm{H}^{+}\right)$.

N-(2-(2-(2-Chloro-4-methylpyridin-3-ylamino)-2-oxoethylthio)benzo[d]thiazol-6-yl)-2-methoxybenzamide (7r). Yellow powder, yield 77\%, purity $90.0 \%$, mp $166.5-168.1{ }^{\circ} \mathrm{C} ;{ }^{1} \mathrm{H}-\mathrm{NMR}$ (DMSO- $\left.d_{6}\right) \delta: 2.22(\mathrm{~s}, 3 \mathrm{H}$ ), $3.90(\mathrm{~s}, 3 \mathrm{H}), 4.41(\mathrm{~s}, 2 \mathrm{H}), 7.08(\mathrm{t}, J=7.2 \mathrm{~Hz}, 1 \mathrm{H}), 7.19(\mathrm{~d}, J=8.4 \mathrm{~Hz}, 1 \mathrm{H}), 7.34(\mathrm{~d}, J=4.8 \mathrm{~Hz}, 1 \mathrm{H})$, 7.52 (t, $J=7.8 \mathrm{~Hz}, 1 \mathrm{H}), 7.64(\mathrm{~d}, J=7.2 \mathrm{~Hz}, 1 \mathrm{H}), 7.69$ (d, $J=8.8 \mathrm{~Hz}, 1 \mathrm{H}), 7.79(\mathrm{~d}, J=8.8 \mathrm{~Hz}, 1 \mathrm{H})$, $8.19(\mathrm{~d}, J=4.8 \mathrm{~Hz}, 1 \mathrm{H}), 8.55(\mathrm{~s}, 1 \mathrm{H}), 10.24(\mathrm{~s}, 1 \mathrm{H}), 10.37(\mathrm{~s}, 1 \mathrm{H}) ;{ }^{13} \mathrm{C}-\mathrm{NMR}\left(\mathrm{DMSO}-d_{6}\right) \delta: 17.84$, 39.51, 55.87, 111.91, 111.97, 119.22,120.49, 120.92, 124.76, 129.65, 130.51, 132.11, 135.47, 135.96, 147.05, 148.70, 148.76, 156.48, 164.03, 164.61, 165.67; HRMS (ESI) $m / z$ : Calcd. for $\mathrm{C}_{23} \mathrm{H}_{19} \mathrm{ClN}_{4} \mathrm{O}_{3} \mathrm{~S}_{2}$ 500.0123; Found: $500.0124\left(\mathrm{M}-\mathrm{H}^{+}\right)$.

2-Methoxy-N-(2-(2-oxo-2-(pyrimidin-2-ylamino)ethylthio)benzo[d] thiazol-6-yl)benzamide (7s). Yellow powder, yield 70\%, purity $90.0 \%, \mathrm{mp} 169.2-171.8{ }^{\circ} \mathrm{C} ;{ }^{1} \mathrm{H}-\mathrm{NMR}$ (DMSO- $\left.d_{6}\right) \delta$ : $3.90(\mathrm{~s}, 3 \mathrm{H}), 4.46(\mathrm{~s}, 2 \mathrm{H}$ ), $7.08(\mathrm{t}, J=7.4 \mathrm{~Hz}, 1 \mathrm{H}), 7.18-7.24(\mathrm{~m}, 2 \mathrm{H}), 7.52(\mathrm{t}, J=7.6 \mathrm{~Hz}, 1 \mathrm{H}), 7.64-7.69(\mathrm{~m}, 2 \mathrm{H}), 7.78(\mathrm{~d}$, $J=8.8 \mathrm{~Hz}, 1 \mathrm{H}), 8.54(\mathrm{~s}, 1 \mathrm{H}), 8.69(\mathrm{~d}, J=8.4 \mathrm{~Hz}, 2 \mathrm{H}), 10.35(\mathrm{~s}, 1 \mathrm{H}), 11.04(\mathrm{~s}, 1 \mathrm{H}) ;{ }^{13} \mathrm{C}-\mathrm{NMR}$ (DMSO- $d_{6}$ ) $\delta: ~ 39.46,55.86,111.84,111.95,119.17,120.48,121.00,124.67,129.68,132.12,135.38$, $135.90,148.80,156.48,157.37,158.41,164.45,164.55,166.18$; HRMS (ESI) $\mathrm{m} / z$ : Calcd. for $\mathrm{C}_{21} \mathrm{H}_{17} \mathrm{~N}_{5} \mathrm{O}_{3} \mathrm{~S}_{2}$ 452.0846; Found: $452.0821\left(\mathrm{M}-\mathrm{H}^{+}\right)$.

2-Methoxy-N-(2-(2-oxo-2-(thiazol-2-ylamino)ethylthio)benzo[d]thiazol-6-yl)benzamide (7t). Yellow powder, yield 68\%, purity 92.0\%, mp 237.7-239.1 ${ }^{\circ} \mathrm{C} ;{ }^{1} \mathrm{H}-\mathrm{NMR}$ (DMSO- $\left.d_{6}\right) \delta$ : $3.90(\mathrm{~s}, 3 \mathrm{H}), 4.47(\mathrm{~s}, 2 \mathrm{H}$ ), $7.08(\mathrm{t}, J=7.4 \mathrm{~Hz}, 1 \mathrm{H}), 7.19(\mathrm{~d}, J=8.4 \mathrm{~Hz}, 1 \mathrm{H}), 7.25(\mathrm{~d}, J=3.6 \mathrm{~Hz}, 1 \mathrm{H}), 7.52$ (t, $J=6.4 \mathrm{~Hz}, 2 \mathrm{H})$, 7.64-7.69 (m, 2H), $7.77(\mathrm{~d}, J=8.8 \mathrm{~Hz}, 1 \mathrm{H}), 8.54(\mathrm{~s}, 1 \mathrm{H}), 10.36(\mathrm{~s}, 1 \mathrm{H}), 12.55(\mathrm{~s}, 1 \mathrm{H}) ;{ }^{13} \mathrm{C}-\mathrm{NMR}$ $\left(\mathrm{DMSO}-d_{6}\right) \delta: 39.37,56.37,112.39,112.47,114.32,119.71,120.98,121.54,125.24,130.15,132.60$, 135.95, 136.50, 138.24, 149.19, 156.97, 158.27, 164.38, 165.07, 166.18; HRMS (ESI) $\mathrm{m} / z$ : Calcd. for $\mathrm{C}_{20} \mathrm{H}_{16} \mathrm{~N}_{4} \mathrm{O}_{3} \mathrm{~S}_{3} 457.0458$; Found: $457.0457\left(\mathrm{M}-\mathrm{H}^{+}\right)$.

\subsection{Cell Culture}

Cell lines HepG2, HeLa, HCT116, SW620, SKOV-3 and MDA-MB-468 were maintained in Dulbecco's modified Eagle medium (DMEM) containing 10\% fetal bovine serum (FBS), penicillin $(100 \mathrm{U} / \mathrm{mL})$ and streptomycin $(10 \mathrm{mg} / \mathrm{L})$. Cell lines BT474, A431 and SKRB-3 were maintained in Dulbecco's modified Eagle medium (DMEM) containing 20\% fetal bovine serum (FBS), penicillin (100 U/mL) and streptomycin (10 mg/L). Cell lines H460, PC-3, A549, SW480, and BxPC-3 were 
maintained in RPMI 1640 containing 10\% FBS, penicillin (100 U/mL) and streptomycin (10 mg/L). Cells were grown in a $5 \% \mathrm{CO}_{2}$ incubator at $37^{\circ} \mathrm{C}$.

\subsection{Cell Proliferation Assay (MTT Assay)}

Cells (3-5 $\times 10^{3} /$ well) were seeded in $200 \mu \mathrm{L}$ of medium/well in 96-well plates (Costar Corning, Rochester, NY, USA) and cultured for $24 \mathrm{~h}$. The compounds dissolved in dimethylsulfoxide (DMSO) were added to final concentration of $40 \mu \mathrm{M}, 20 \mu \mathrm{M}, 10 \mu \mathrm{M}, 5 \mu \mathrm{M}, 2.5 \mu \mathrm{M}, 1.25 \mu \mathrm{M}$, respectively. Compounds 1 and 2 were used as positive control. After $48 \mathrm{~h}$ exposure, a volume of $10 \mu \mathrm{L}$ of $10 \mathrm{mg} / \mathrm{mL}$ 3-(4,5-dimethylthiazol-2-yl)-2,5-diphenyltetrazolium bromide (MTT) was added per well and incubated for another $4 \mathrm{~h}$ at $37{ }^{\circ} \mathrm{C}$, then the supernatant fluid was removed and DMSO $(150 \mu \mathrm{L})$ was added $150 \mu \mathrm{L} /$ well to dissolve formazan crystals for 15-20 min. The light absorptions (OD) were measured at $570 \mathrm{~nm}$ with SpectraMAXM5 microplate spectrophotometer (Molecular Devices). The effect of compounds on tumor cells viability was expressed by $\mathrm{IC}_{50}$ of each cell line. Values shown are the \% viability $v s$. ctrl $+\mathrm{SD}, \mathrm{n}=$ six independent experiments in triplicate.

\subsection{Apoptosis Analysis by Flow Cytometry (FCM)}

Cells were seeded into $1 \mathrm{~mL}$ of medium/well cell in 6-well plates (Costar Corning) culture bottles at $10 \times 10^{6}$ cells $24 \mathrm{~h}$ before treatment. Then cells were treated with compound 7e for $24 \mathrm{~h}: 0 \mu \mathrm{M}$ (control), $0.625 \mu \mathrm{M}, 1.25 \mu \mathrm{M}, 2.5 \mu \mathrm{M}$. After $24 \mathrm{~h}$ incubation, floating and adherent cells were collected, washed three times with PBS ( $\mathrm{pH} 7.4)$ and fixed for $24 \mathrm{~h}$ with cool alcohol at $4{ }^{\circ} \mathrm{C}$. $1 \mathrm{~mL}$ cell suspension $\left(10^{6} / \mathrm{mL}\right)$ was washed three times with cooled PBS, treated with RNase for $30 \mathrm{~min}$ at $37{ }^{\circ} \mathrm{C}$, stained it with PI for $30 \mathrm{~min}$ at $37{ }^{\circ} \mathrm{C}$ in a dark environment, and taken for flow cytometry analysis.

\section{Conclusions}

In conclusion, a series of novel benzothiazole-2-thiol derivatives were synthesized and their anti-proliferative activities were evaluated in vitro. The results showed that the pyridinyl-2-amine linked benzothiazole-2-thiol compounds $\mathbf{7 d}, \mathbf{7 e}, \mathbf{7} \mathbf{f}$ and $\mathbf{7 i}$ exhibited increased anticancer activities compared with compounds $\mathbf{1}$ and $\mathbf{2}$ on the three human cancer cells (SW480, HeLa and HepG2). Further studies showed that they displayed potent and broad-spectrum anti-proliferative activities against other human cancer cell lines. Among these compounds, compound 7e displayed the most potent anticancer activity on SKRB-3 $\left(\mathrm{IC}_{50}=1.2 \mathrm{nM}\right), \mathrm{SW} 620\left(\mathrm{IC}_{50}=4.3 \mathrm{nM}\right), \mathrm{A} 549\left(\mathrm{IC}_{50}=44 \mathrm{nM}\right)$ and HepG2 $\left(\mathrm{IC}_{50}=48 \mathrm{nM}\right)$. The results of flow cytometry analysis indicated that compound 7e induce apoptosis in HepG2 cancer cells on a concentration-dependent manner. These data suggested that compound 7e may be powerful tumor growth inhibitors via apoptosis as novel anticancer agents and be worth being further investigated as a potential of anticancer agent. 


\section{Acknowledgements}

This work was supported by National Science and Technology Major Project of China (2009ZX09103-132). The authors gratefully thank Yong-Qiu Mao (State Key Laboratory of Biotherapy, Huaxi Hospital, Sichuan University) for helpful technical assistance and discussion in the flow cytometry analysis.

\section{References and Notes}

1. Quiroga, J.; Hernandez, P.; Insuasty, B.; Abonıa, R.; Cobo, J.; Sanchez, A.; Nogueras, M.; Low, J.N. Control of the reaction between 2-aminobenzothiazoles and Mannich bases. Synthesis of pyrido[2,1-b][1,3]benzothiazoles versus $[1,3]$ benzothiazolo[2,3-b]quinazolines. J. Chem. Soc. Perkin Trans. I 2002, 1, 555-559.

2. Kok, S.H.L.; Chui, C.H.; Lam, W.S.; Chen, J.; Lau, F.Y.; Wong, R.S.M.; Cheng, G.Y.M.; Lai, P.B.S.; Leung, R.W.T.; Tang, J.C.O.; et al. Synthesis and structure evaluation of a novel cantharimide and its cytotoxicity on SK-Hep-1 hepatoma cells. Bioorg. Med. Chem. Lett. 2007, 17, 1155-1159.

3. Kok, S.H.L.; Chui, C.H.; Lam, W.S.; Chen, J.; Lau, F.Y.; Wong, R.S.M.; Cheng, G.Y.M.; Tang, W.K.; Teo, I.T.N.; Cheung, F.; et al. Apoptogenic activity of a synthetic cantharimide in leukaemia: Implication on its structural activity relationship. Int. J. Mol. Med. 2006, 18, 1217-1221.

4. Kok, S.H.L.; Gambari, R.; Chui, C.H.; Yuen, M.C.W.; Lin, E.; Wong, R.S.M.; Lau, F.Y.; Cheng, G.Y.M.; Lam, W.S.; Chan, S.H.; et al. Synthesis and anti-cancer activity of benzothiazole containing phthalimide on human carcinoma cell lines. Bioorg. Med. Chem. 2008, 16, 3626-3631.

5. Song, E.Y.; Kau, R.N.; Park, M.Y.; Jin, Y.L.; Lee, K.; Kim, G.; Lee, K.Y.; Yang, J.S.; Shin, J.H.; Nam, K.Y.; et al. Synthesis of amide and urea derivatives of benzothiazole as Raf-1 inhibitor. Eur. J. Med. Chem. 2008, 43, 1519-1524.

6. Wang, Z.; Shi, X.H.; Wang, J.; Zhou, T.; Xu, Y.Z.; Huang, T.T.; Li, Y.F.; Zhao, Y.L.; Yang, L.; Yang, S.Y.; et al. Synthesis, structure-activity relationships and preliminary antitumor evaluation of benzothiazole-2-thiol derivatives as novel apoptosis inducers. Bioorg. Med. Chem. Lett. 2011, $21,1097-1101$.

7. Guo, X.Z.; Shi, L.; Wang, R.; Liu, X.X.; Li, B.G.; Lu, X.X. Synthesis and biological activities of novel nonpeptide angiotensin II receptor antagonists based on benzimidazole derivatives bearing a heterocyclic ring. Bioorg. Med. Chem. Lett. 2008, 16, 10301-10310.

8. Liu, Y.; Gerald, N.L. Activation of heterocyclic amines by combinations of prostaglandin $\mathrm{H}$ synthase-1 and -2 with $N$-acetyltransferase 1 and 2. Cancer Lett. 1998, 133, 115-123.

9. Peng, F.; Wei, Y.Q.; Tian, L.; Yang, L.; Zhao, X.; Lu, Y.; Mao, Y.Q.; Kan, B.; Lei, S.; Wang, G.S.; et al. Induction of apoptosis by norcantharidin in human colorectal carcinoma cell lines: Involvement of the CD95 receptor/ligand. Cancer Res. Clin. Oncol. 2002, 128, 223-230.

10. Baraldi, P.G.; Preti, D.; Tabrizi, M.A.; Fruttarolo, F.; Saponaro, G.; Baraldi, S.; Romagnoli, R.; Moorman, A.R.; Gessi, S.; Varani, K.; et al. Hybrid molecules between distamycin A and active moieties of antitumor agents. Bioorg. Med. Chem. 2007, 15, 17-35. 
11. Musso, D.L.; Cochran, F.R.; Kelley, J.L.; McLean, E.W.; Selph, J.L.; Rigdon, G.C.; Orr, G.F.; Davis, R.G.; Cooper, B.R.; Styles, V.L.; et al. Indanylidenes. 1. Design and synthesis of (E)-2-(4,6-difluoro-1-indanylidene)acetamide, a potent, centrally acting muscle relaxant with antiinflammatory and analgesic activity. J. Med. Chem. 2003, 46, 399-408.

Sample Availability: Samples of the compounds $7 \mathbf{a}-\mathbf{t}$ are available from the authors.

(C) 2012 by the authors; licensee MDPI, Basel, Switzerland. This article is an open access article distributed under the terms and conditions of the Creative Commons Attribution license (http://creativecommons.org/licenses/by/3.0/). 